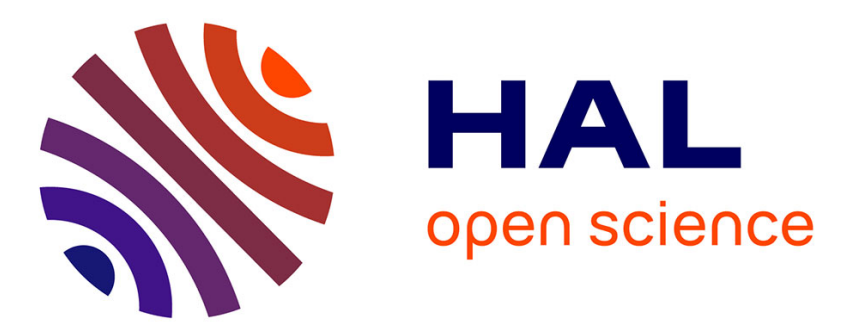

\title{
Charge Dynamics and Optolectronic Properties in HgTe Colloidal Quantum Wells
}

Clément Livache, Eva Izquierdo, Bertille Martinez, Marion Dufour, Debora Pierucci, Sean Keuleyan, Hervé Cruguel, Loic Becerra, Jean Louis Fave, Hervé Aubin, et al.

\section{To cite this version:}

Clément Livache, Eva Izquierdo, Bertille Martinez, Marion Dufour, Debora Pierucci, et al.. Charge Dynamics and Optolectronic Properties in HgTe Colloidal Quantum Wells. Nano Letters, 2017, 17 (7), pp.4067-4074. 10.1021/acs.nanolett.7b00683 . hal-01541341

\section{HAL Id: hal-01541341 https://hal.sorbonne-universite.fr/hal-01541341}

Submitted on 19 Jun 2017

HAL is a multi-disciplinary open access archive for the deposit and dissemination of scientific research documents, whether they are published or not. The documents may come from teaching and research institutions in France or abroad, or from public or private research centers.
L'archive ouverte pluridisciplinaire HAL, est destinée au dépôt et à la diffusion de documents scientifiques de niveau recherche, publiés ou non, émanant des établissements d'enseignement et de recherche français ou étrangers, des laboratoires publics ou privés. 


\title{
Charge dynamics and optolectronic properties in HgTe colloidal quantum wells
}

\author{
Clément Livache ${ }^{1,2}$, Eva Izquierdo ${ }^{2}$, Bertille Martinez ${ }^{1}$, Marion Dufour ${ }^{2}$, Debora Pierucci ${ }^{3}$, \\ Sean Keuleyan ${ }^{4}$, Hervé Cruguel ${ }^{1}$, Loic Becerra ${ }^{1}$, Jean Louis Fave ${ }^{1}$, Hervé Aubin ${ }^{2}$, Abdelkarim \\ Ouerghi $^{5}$, Emmanuelle Lacaze ${ }^{1}$, Mathieu G. Silly ${ }^{6}$, Benoit Dubertret ${ }^{2}$, Sandrine Ithurria ${ }^{2}$, \\ Emmanuel Lhuillier ${ }^{1 *}$ \\ ${ }^{1}$ Sorbonne Universités, UPMC Univ. Paris 06, CNRS-UMR 7588, Institut des NanoSciences de \\ Paris, 4 place Jussieu, 75005 Paris, France \\ ${ }^{2}$ Laboratoire de Physique et d'Etude des Matériaux, ESPCI-ParisTech, PSL Research \\ University, Sorbonne Université UPMC Univ Paris 06, CNRS, 10 rue Vauquelin 75005 Paris, \\ France. \\ ${ }^{3}$ Institut Néel, CNRS-UJF, BP 166, 38042 Grenoble Cedex 9, France \\ ${ }^{4}$ Voxtel, Inc., University of Oregon, CAMCOR, 1241 University of Oregon, Eugene, OR 97403, \\ USA \\ ${ }^{5}$ Laboratoire de Photonique et de Nanostructures (CNRS-LPN), Route de Nozay, 91460 \\ Marcoussis, France \\ ${ }^{6}$ Synchrotron-SOLEIL, Saint-Aubin, BP48, F91192 Gif sur Yvette Cedex, France
}

\begin{abstract}
We investigate the electronic and transport properties of HgTe 2D colloidal quantum wells. We demonstrate that the material can be made $p$ or $n$-type depending on the capping ligands. In addition to the control of majority carrier type, the surface chemistry also strongly affects the photoconductivity of the material, . These transport measurements are correlated with the electronic structure determined by high resolution X-ray photoemission. We attribute the change of majority carriers to the strong hybridization of an $n$-doped HgS layer resulting from capping of the HgTe nanoplatelets by $\mathrm{S}^{2-}$ ions. We further investigate the gate and temperature dependence of the photoresponse and its dynamics. We show that the photocurrent rise and fall times can be tuned from $100 \mu$ s to $1 \mathrm{~ms}$ using the gate bias. Finally, we use time-resolved photoemission spectroscopy as a probe of the transport relaxation to determine if the observed dynamics are limited by a fundamental process such as trapping. These pump probe surface photovoltage measurements show an even faster relaxation in the $100 \mathrm{~ns}$ to $500 \mathrm{~ns}$ range, which suggests that the current performances are rather limited by geometrical factors.
\end{abstract}

Keywords : HgTe, nanocrystals, 2D nanoplatelets, photoresponse, carrier dynamics

*To whom correspondence should be addressed. E-mail: el@insp.upmc.fr 


\section{Introduction}

Colloidal quantum dots (CQD) are attracting a large interest because of their tunable optical properties from UV to $\mathrm{THz}^{1-3}$ Their integration into optoelectronic devices ${ }^{4}$ is seen as the next challenge after their successful use as the newest generation of phosphor for displays. In this perspective, addressing the infrared (IR) is of utmost interest since current technologies are not able to combine high performance with low cost. CQD can combine the stability of inorganic material with the ease of processing of organic electronic materials, which makes them a potential candidate for the design of the next generation of IR devices. To obtain IR optical transitions within CQD, narrow band gap materials have to be used ${ }^{5,6}$. In the IR, mercury chalcogenides ${ }^{7,8}(\mathrm{HgX})$ have so far been the most successful materials ${ }^{9}$ (for a recent review see ref ${ }^{10}$ ), because of the semimetal nature of bulk $\mathrm{HgTe}$ and HgSe. For near-IR, lead chalcogenides such as PbS are certainly the most investigated material ${ }^{11}$. However, PbS CQD used for solar cells are small CQD and surface traps strongly limit their overall performances. Traps in PbS lead to a broadening of the photoluminescence (PL) linewidth ${ }^{12}$ and limit the open circuit voltage of solar cells ${ }^{13}$. To obtain improved optical features, the use of 2D nanoplatelets (NPL) has led to some success because their growth process prevents ${ }^{14}$ roughness in the confined dimension. This results in the absence of inhomogeneous broadening for these materials ${ }^{15}$. Up to recently, this result was only obtained with cadmium chalcogenide $(\mathrm{CdX})$ compounds ${ }^{14,16}$. CdX NPL present exceptionally narrow optical features with a linewidth below $2 \mathrm{k}_{\mathrm{b}} \mathrm{T}$. Recently, Izquierdo et al. ${ }^{17}$ proposed the synthesis of $\mathrm{HgX}$ NPL via cation exchange from CdX NPL, while preserving exceptional control of the optical properties. They demonstrated near IR (800-900 $\mathrm{nm}$ ) emission with PL linewidth as narrow as $60 \mathrm{meV}$; half that typically reported for PbS CQD. Consequently, HgX NPL look promising for the design of near IR infrared materials.

In this paper, we investigate the transport and electronic properties of HgTe NPL. In the first part, we correlate the effects of surface chemistry on the energy levels and electrical conductivity. We demonstrate that HgTe NPL can be made $p$ or $n$-type using ethanedithiol (EDT) and $\mathrm{S}^{2-}$ capping, respectively. Using $\mathrm{x}$-ray photoemission (XPS) spectroscopy, we attribute the change of the carrier density to a difference in hybridization of the two ligands with the Hg rich surface of the NPL, which results in a shift of the bands with respect to the Fermi level. In the second part of the manuscript, we investigate the carrier dynamics. Two challenges must be addressed to probe the dynamics at the device scale: long time scale dynamics ( $t>$ hopping time) need to be explored, and the method must be compatible with the narrow band gap material. This combination is quite challenging for conventional optical time resolved spectroscopy. Using transient photocurrents, we measure a response time as fast as $100 \mu$ s and we question whether this is a fundamental limit. To answer this, we conduct time resolved photoemission measurements and demonstrate that the transport relaxation occurs on a faster time scale, of the order of 100 ns. This suggests that current performances are limited by geometrical factors rather than physical processes.

\section{Discussion}

To grow HgTe NPL, we first synthesize CdTe NPL following the procedure developed by Pedetti et $a I^{18}$ In a second step, the cadmium cations are exchanged for mercury by exposing the CdTe NPL to mercury ions complexed by oleylamine ${ }^{15}$. After a full exchange of the $\mathrm{Cd}^{2+}$ ions, we obtain 2D HgTe NPL. These NPL have a $1.1 \mathrm{~nm}$ thickness ${ }^{17}$ and their lateral dimension exceeds $100 \mathrm{~nm}$, see Figure $1 \mathrm{a}$. After the cation exchange, the excitonic feature shifts from $500 \mathrm{~nm}$ to $880 \mathrm{~nm}$, as shown in Figure $1 \mathrm{~b}$. Figure $1 \mathrm{c}$ shows a photoluminescence decay of tens of $\mathrm{ns}$; fast for near IR emitters which are typically in the $\mu$ s range. 

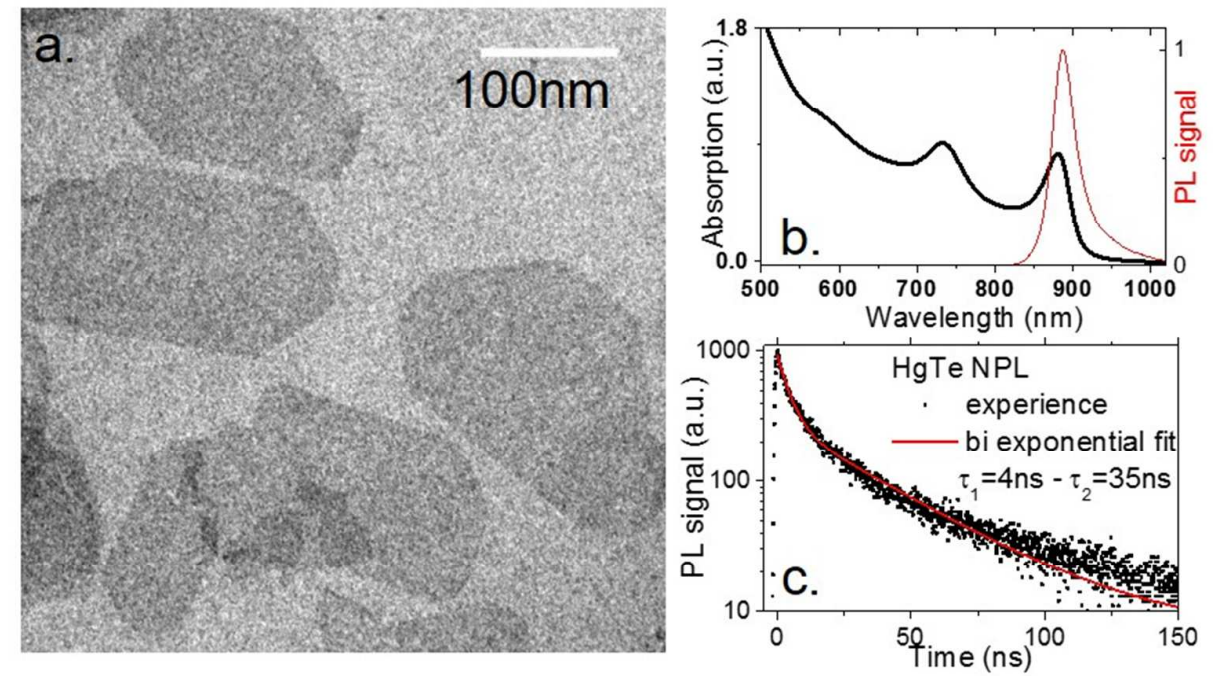

Figure 1 a. Transmission electron microscopy image of HgTe NPL. b. Absorption and photoluminescence spectrum of HgTe NPL. c. Time resolved photoluminescence measurement for a solution of HgTe NPL

Thin films by drop- or spin-casting are electrically insulating as-deposited. To explore charge transport and photoconductivity, the ligands on the NPL are exchanged after depositing into a film. Ethanedithiol $(E D T)^{19}$ and sulfide ions ${ }^{20}\left(\mathrm{~S}^{2-}\right)$ are used for their high affinity for mercury and small size, giving strong inter-NPL coupling. After ligand exchange, the absorption spectrum of the NPL is redshifted to $950 \mathrm{~nm}(\approx 1.35 \mathrm{eV})$, consistent with increased delocalization of the wavefunction, but indicating that confinement remains strong and individual platelets are preserved. This conclusion is further confirmed by scanning and transmission electron microscopy (SEM and TEM) imaging after the ligand exchange, see figure S1. Thin films (roughly $100 \mathrm{~nm}$ thick) are dropcast on interdigitated electrodes with $20 \mu \mathrm{m}$ spacing. Following ligand exchange, an ion gel ${ }^{21,22}$ made of $\mathrm{LiClO}_{4}^{-}$in polyethylene glycol (PEG) and gate electrode are added, forming an electrolytic transistor. Details of the preparation are provided in the supporting information. The use of this quasi-solid electrolyte gating enables injection of a large carrier density and operation in air. Ions can permeate the film between NPL, ${ }^{22}$ and thick films can therefore be gated, which is useful to design phototransistors ${ }^{23}$.

HgTe NPL films capped by these two ligands show very different electrical behaviors. With EDT the film conductance rises as holes are injected under negative gate bias, corresponding to a $p$-type channel, see Figure $2 \mathrm{a}$. In the case of $\mathrm{S}^{2-}$ capping the conductance rises as electrons are injected under positive gate bias, indicating an n-type channel, shown in Figure $2 \mathrm{~b}$. From the bias of the charge neutrality point we can determine the carrier density, using a reasonable assumption for the electrolytic gate capacitance $\left(C \approx 2 \mu \mathrm{F} / \mathrm{cm}^{-2}\right)^{24}$. We estimate in the EDT-exchanged film, a hole density of $2.5 \times 10^{12} \mathrm{~cm}^{-3}$ and in the $\mathrm{S}^{2-}$ exchanged films, an electron density of $4.8 \times 10^{12} \mathrm{~cm}^{-3} . \mathrm{In}$ fact, both treatments give ambipolar transport, though the majority carrier mobilities are at least 10 times higher than that of the minority carrier. Photocurrent is observed in both cases, but is greater in the EDT exchanged films. Under $5 \mathrm{~V}$ bias at room temperature, EDT exchanged films showed a responsivity of $100 \mathrm{~mA} \mathrm{~W}-1$ to $808 \mathrm{~nm}$ light, while the responsivity of the $\mathrm{S}^{2-}$ exchanged film was 20 $\mathrm{mA} \mathrm{W}^{-1}$, see Figure $2 \mathrm{c}$. These observations highlight a need for a better understanding of the electronic structure of the HgTe NPL after their functionalization by the ligands. 

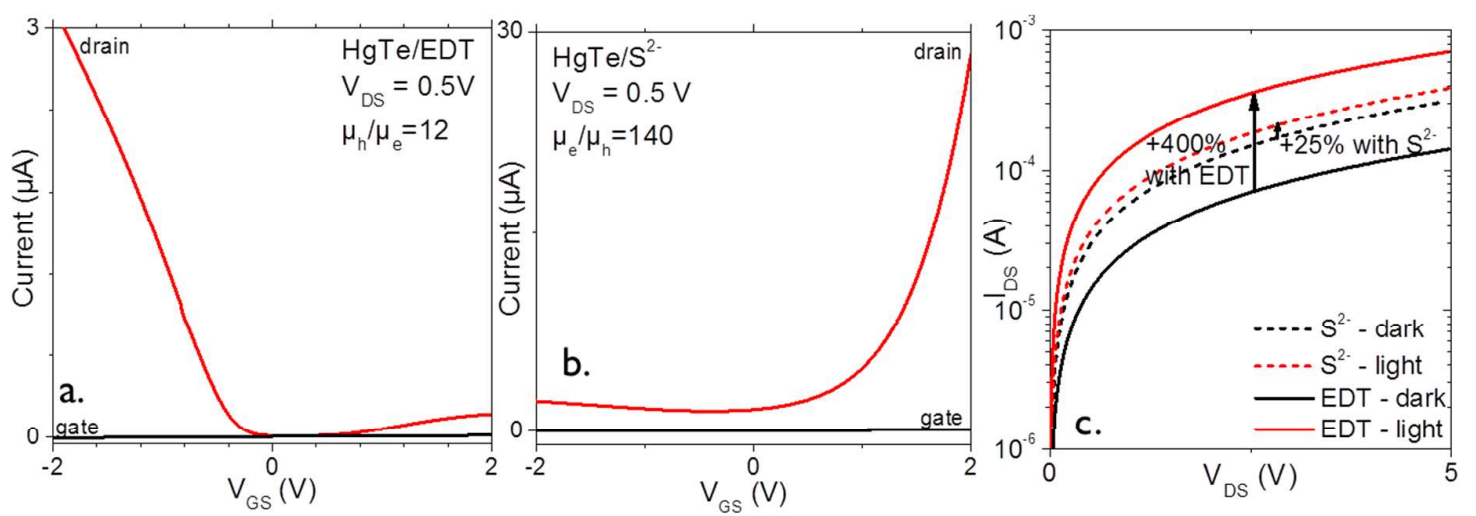

Figure 2 Transfer curves (drain current as a function of gate bias) for a HgTe NPL thin film capped with EDT (a) or with $\mathrm{S}^{2-}$ (b). c. IV curve with and without illumination using an $808 \mathrm{~nm}$ laser diode with a $0.15 \mathrm{~W} \mathrm{~cm}^{-2}$ irradiance.

We conducted XPS measurements on the Tempo beamline of the Soleil synchrotron; see section 3 of the $\mathrm{SI}$ and the associated figures. XPS of the $\mathrm{Hg}$ core level reveals only one significant contribution to the $\mathrm{Hg} 4 \mathrm{f}$ peak level. Figure 3 a shows that as the capping ligand is switched from EDT to $\mathrm{S}^{2-}$, the peaks are shifted to lower binding energies by $200 \mathrm{meV}$, indicating that the $\mathrm{Hg}$ state is richer in electron density. To further confirm this, we measured the secondary electron cut-off as well as the top of the valence band for both ligand treatments. Details are given in section 3 of the SI. From these measurements, we determine the material work function and absolute energy (vs vacuum) of the valence band. With the band gap obtained from the optical absorption, the effective absolute energy spectrum for HgTe NPL is given in Figure 3b. In the case of EDT capping, the Fermi level is below the middle of the band gap, while for $\mathrm{S}^{2-}$ capping, it is close to the conduction band. This is consistent with their respective $p$ and $n$-type character observed in transport measurements.

The work function is largely unaffected by the ligand exchange $(\approx 4.35 \mathrm{eV})$. In $\mathrm{HgSe}^{25}$ and $\mathrm{PbS}^{26}$, doping levels are strongly dependent on the surface ligands whereas here, the change of population in our NPL is not related to an effect of surface dipoles, since the latter would lead to a shift of the vacuum level. In the dipole induced energy level shifts, surface bound $\mathrm{S}^{2-}$ pushes the states up, leading to $\mathrm{p}$-type doping as observed for $\mathrm{HgSe}^{25}$. In contrast, we observe $\mathrm{n}$-type doping with $\mathrm{S}^{2-}$ treatment. Thus the $\mathrm{S}^{2-}$ is not simply contributing to a surface dipole but hybridizing with the HgTe states forming an $n$-doped $\mathrm{HgS}$ layer ${ }^{27}$. This change of doping level with ligand also explains the observed difference of photoconductivity. With a higher doping level, the $\mathrm{S}^{2-}$ capped HgTe NPL film has a higher dark current which reduces the relative light modulation. In addition, the Fermi level is very close to the conduction band edge and high occupation of the conduction band state may partially bleach the interband transition and reduce absorption. 

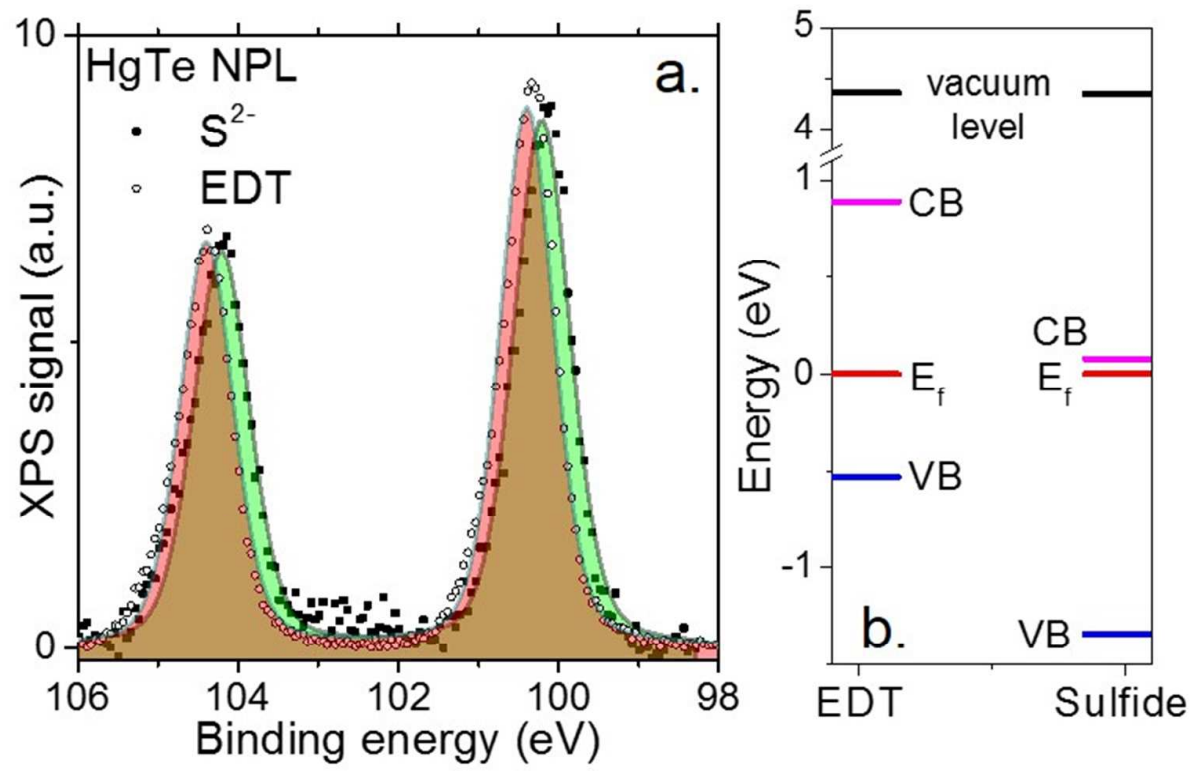

Figure 3a. XPS spectrum of the Hg $4 f$ core level for a film of HgTe NPL capped with EDT and $S^{2-} b$. Electronic spectrum of HgTe NPL caped with $S^{2-}$ and EDT. The band gap is determined from UV-Vis. The Fermi level and valence band energies are determined by photoemission.

Infrared photodetectors are typically operated at reduced temperature to suppress the dark current from thermally excited carriers, as noise originating in the dark current is often the limiting factor in photodetector performance. This is especially the case in the infrared at room temperature operation. The temperature dependence of the dark current provides information on the origin of thermal carriers, where intrinsic interband generation follows a roughly Arrhenius behavior with an activation energy of half the band gap. Smaller activation is an indication of extrinsic carriers and a Fermi level that is closer to one of the bands, or that conductivity is limited by a temperature dependent transport mechanism. Figure 4a shows the current as a function of temperature with and without illumination (60 mW at $808 \mathrm{~nm}$ ). As the photocurrent is at least 2 orders of magnitude greater than the dark current over this temperature range, the total current under illumination is approximately equal to the photocurrent. An Arrhenius fit of the high temperature $\left(250-330^{\circ} \mathrm{C}\right)$ dark conductance gives an activation energy of $\approx 250 \mathrm{meV}$ (see Figure S3), only half of the difference between the valence band and fermi level. This suggests the presence of shallow trap states near the valence band, as discussed later. The photocurrent shows a smaller temperature dependence, attributed to activated mobility. Because the dark current is reduced more quickly than the photocurrent on cooling, better signal to noise is possible as long as the device is limited by dark current noise. This is highlighted in Figure $4 \mathrm{~d}$, which shows $I_{\text {light }} / /_{\text {dark }}$ as a function of temperature. 

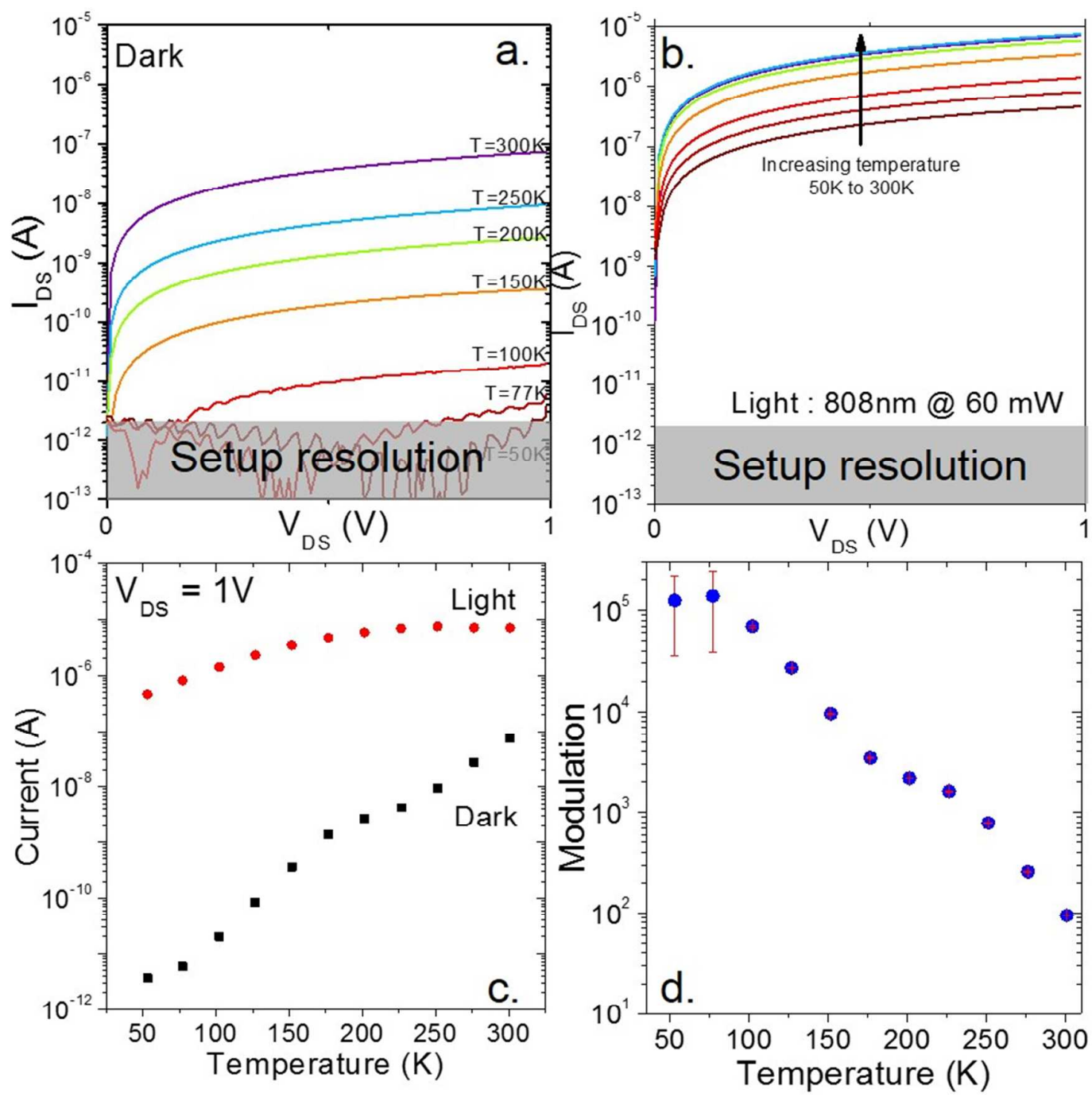

Figure 4a. IV curves at different temperatures for a thin film of HgTe NPL (EDT capping) in dark. b. IV curves at different temperatures for a thin film of HgTe NPL (EDT capping) under illumination by an $808 \mathrm{~nm}$ laser. c. Temperature dependence of the current and with and without illumination, measured at $1 \mathrm{~V}$ for a thin film of HgTe NPL (EDT capping). $d$. Evolution of the ratio of the current under illumination divided by the dark current as a function of temperature for a thin film of $\mathrm{HgTe}$ NPL (EDT capping). The error bars result from the dark current being limited by the setup resolution at low temperature.

The effect of the gate on the photocurrent is then investigated. It has been previously demonstrated that electrolyte gating can be used to enhance the photoresponse of CdSe/CdS NPL films ${ }^{22,23}$. In such wide band gap materials, intrinsic carrier densities are small, and the cost of increasing the dark current slightly is outweighed by enhancements in the photoresponse coming from trap-filling. For narrower band gap materials used for IR detection, reducing the dark current is a priority to limit noise ${ }^{28}$. The gate can be used to reduce the majority carrier density and the associated dark current. Figure $5 \mathrm{~b}$ shows the ratio of the current under illumination to the dark current as a function of gate bias. At positive gate potential, electrons are injected into the film and the ratio increases. From the activation energy of the hole traps ( $E_{T}-E_{V} \approx 250 \mathrm{meV}$ ) and assuming a $2 \mathrm{D}$ density of states for the 
NPL given by $\frac{m^{*}}{\pi \hbar^{2}}\left(m^{*}=0.2 m_{0}\right)^{29}$, we can estimate the thermally activated hole carrier density ${ }^{30}$ to be $p=\frac{m^{*}}{\pi \hbar^{2}} E_{C} \exp \left(-\frac{E_{T}-E_{V}}{k_{b} T}\right) \approx 7 x 10^{13} \mathrm{~cm}^{-2}$ at room temperature, with $\mathrm{E}_{\mathrm{c}}$ the confinement energy $(\approx 1.35 \mathrm{eV})$. This must be compared with the steady-state photogenerated carrier density given by $p_{\text {light }} \approx F \tau \alpha L=2.6 \times 10^{13} \mathrm{~cm}^{-2}$, where $\mathrm{F}$ is the incident photon flux $\left(90 \mathrm{~mW} \mathrm{~cm} \mathrm{~cm}^{-2} @ 808\right.$ $\left.\mathrm{nm}=3.7 \times 10^{19} \mathrm{~cm}^{-2} \mathrm{~s}^{-1}\right), \tau$ is the minority carrier lifetime, taken as $1 \mathrm{~ms}, \alpha$ is the absorption coefficient at $808 \mathrm{~nm} \approx 7000 \mathrm{~cm}^{-1}$, and L is the NPL thickness $(1 \mathrm{~nm})$. This estimate gives $\frac{\left(p_{\text {light }}+p\right)}{p}=1.4$, which is in the range of $I_{\text {light }} / I_{\text {dark }}$ observed. Performance improvements will require either better surface passivation or a lower operating temperature.
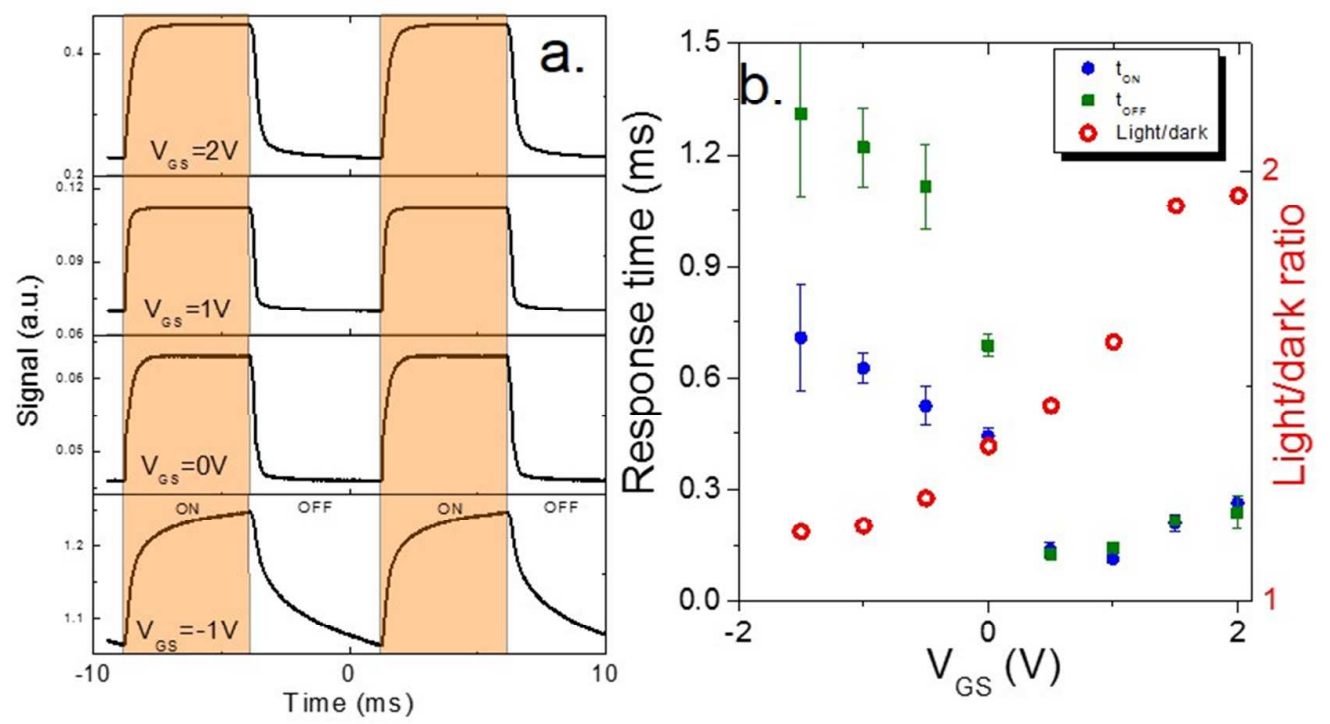

Figure $5 \mathrm{a}$. Trace of the current while the light $\left(808 \mathrm{~nm}\right.$ diode, $\left.90 \mathrm{~mW} \mathrm{~cm}{ }^{-2}\right)$ is turned on and off under different gate bias, at room temperature. $b$. On and off response time to light and the ratio of the current with and without illumination as a function of the gate bias. The error bars are based on the hysteresis when the gate bias is sweeped back and forth.

Applying a gate bias affects the dark current magnitude, but also strongly affects the carrier dynamics. Figure $5 a$ shows the rise and fall of the current when the film is exposed to light under different gate biases. An electronically modulated $808 \mathrm{~nm}$ laser diode is used for illumination and the current is collected using an oscilloscope. The rise and fall times of the device are strongly affected by the Fermi level. Figure $5 b$ shows that the decay tends to be slower than the rise. The overall shape of the gate dependence follows the same trend as the transistor transfer curve (see Figure 2a). The dynamics tend to be slower with higher carrier densities and faster when they are minimized. Figure 2a shows that in the EDT-capped NPL film, electrons have a much lower mobility. Photogenerated holes can move if as long as they do not get trapped in the shallow traps near the valence band. Under negative gate bias, injected holes fill these shallow traps and increase the time spent by the majority carrier within the valence band, which results in the observed change of dynamics in good agreement with previously proposed model ${ }^{22,23}$. Improvement of light over dark current ratio is mainly due to dark current reduction. The response time of the device can be as fast as $100 \mu \mathrm{s}$. It is 
important to identify whether this is the result of physical limitations (traps, etc.), or it can be mad faster.

Carrier dynamics in films of CQD are generally obtained using optical measurements such as time resolved $\mathrm{PL}$, which requires high $\mathrm{PL}$ quantum yields, or transient absorption, which requires a large excitation density to be measurable. ${ }^{31,32}$ Both of these methods are typically limited to short time scales (<ns). Transport in NPL films, however, requires a time scale longer than the hopping time $\tau_{\text {hop }}$, the time for a charge to jump from one NPL to the other. $\tau_{\text {hop }}$ is given by $\tau_{h o p}=\frac{e L^{2}}{6 \mu k_{b} T}$ with $e$ the proton charge, $L$ the hopping length, $\mu$ the carrier mobility and $k_{b} T$ the thermal energy. The thickness of the NPL is $1.1 \mathrm{~nm}$ so we can estimate the hopping distance between NPL to be $1.7 \mathrm{~nm}$ with $\mathrm{S}^{2-}$ and $2.1 \mathrm{~nm}$ with EDT. Mobility is typically around $10^{-3} \mathrm{~cm}^{2} V^{-1} \mathrm{~s}^{-1}$ for EDT capping ${ }^{19}$ and can be as high as $1 \mathrm{~cm}^{2} V^{-1} \mathrm{~s}^{-1}$ for $\mathrm{S}^{2-} \cdot{ }^{20}$ With these values the hopping time spans from the ps to ns range. As a result, time resolved optical measurements mostly probe intradot processes ${ }^{33,34}$ (Auger, radiative recombination...) and are consequently not useful for the investigation of the slower dynamics (ns- $\mu \mathrm{s}$ and ms range) in transport mechanisms.

In addition, transport requires ligand exchange to short ligands, which quenches the PL and introduces non-radiative decay paths. We thus expect different dynamics between PL and transport. To illustrate, the PL decay of the HgTe NPL has been measured to be in the tens of ns range (Figure 1c) while the photocurrent fall times are typically between $100 \mu$ s and 1 ms (Figure 5). Probing carrier dynamics becomes even more challenging as the optical feature of the CQD are shifted toward IR and mid IR, ${ }^{35}$ because of the lower efficiency of optical setups and intrinsically lower $\mathrm{PL}$ yield. Alternative ways to probe carrier relaxation which can be applied to narrow band gap conductive films are therefore necessary.

Time resolved XPS is used here, in a pump probe configuration illustrated in Figure $6 a .{ }^{36,37} 100 \mathrm{~nm}$ thick films, much thinner than the absorption length of several microns are used so that the excitation density is uniform. The sample is pumped with an red $(800 \mathrm{~nm}, 80 \mathrm{fs})$ or a blue $(405 \mathrm{~nm}$, square pulse with 2 ns rise) laser. An XPS spectrum of the $\mathrm{Hg} 4 \mathrm{f}$ region is then acquired every $10 \mathrm{~ns}$. The resolution of the experiment is limited by the analyzer to around $30 \mathrm{~ns}$. A scheme of the experiment is given in Figure 6a. The photoemission spectrum is then fitted and Figure $6 \mathrm{~b}$ and $\mathrm{c}$ show the energy of the peak as a function of time for EDT and $\mathrm{S}^{2-}$ capping, respectively.. 
a.

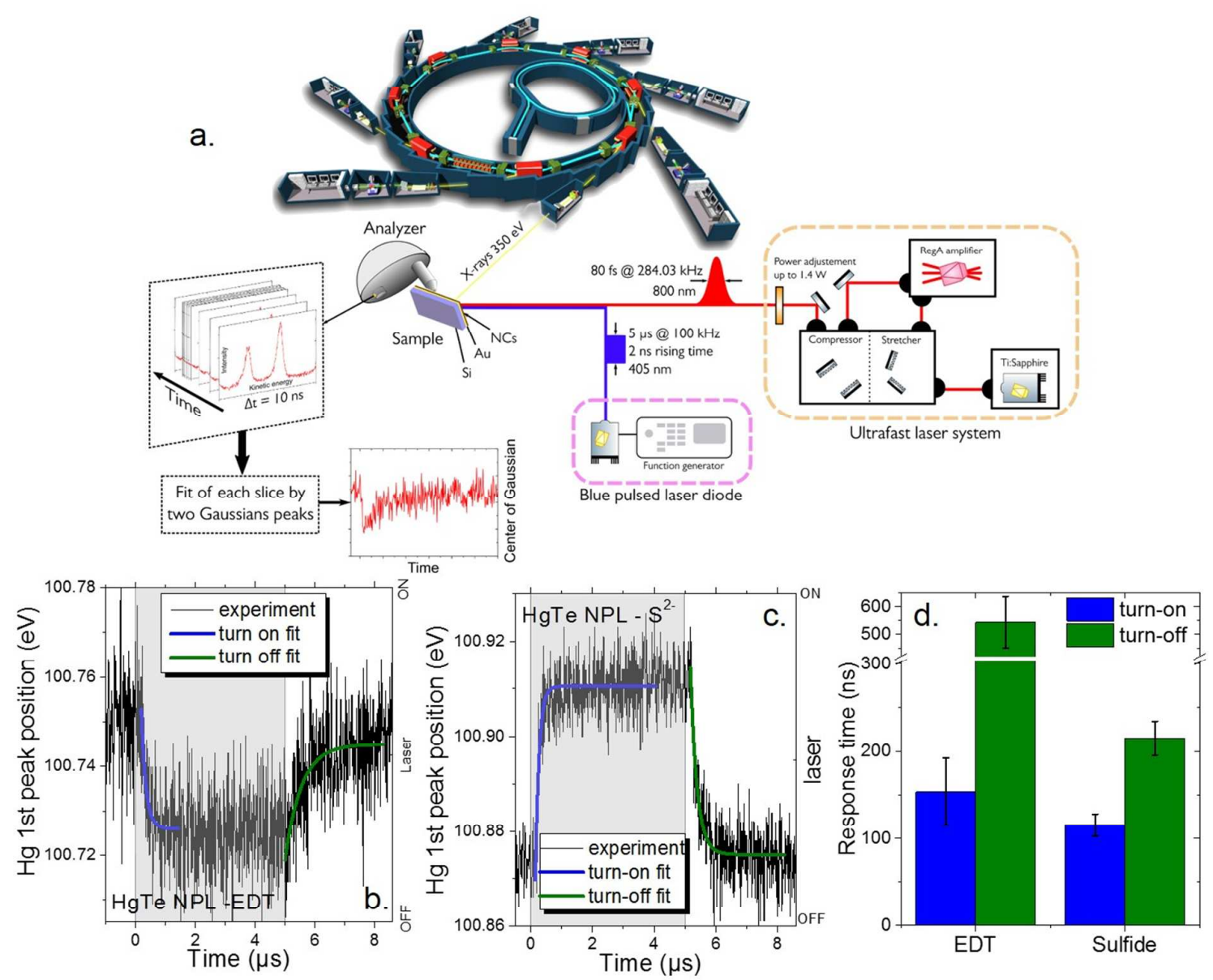

Figure 6 a. Scheme of the time resolved surface photovoltage measurements. Two laser sources can be used: either a $80 \mathrm{fs}$ amplified Ti sapphire laser operated at $800 \mathrm{~nm}$ or a $2 \mathrm{~ns}$ rise time blue (405 $\mathrm{nm}$ ) laser diode. The sample is under ultra high vacuum. $350 \mathrm{eV} X$-rays from the Soleil synchrotron are coincident with the laser spot. Every $10 \mathrm{~ns}$, a photoemission spectrum is acquired. The time resolution of the experiment is $30 \mathrm{~ns}$, limited by the analyzer. These spectra are then fitted by a two Gaussians and the center of the low BE Gaussian is displayed as a function of time. a. Synchrotron diagram from the Soleil website. $b$. and c. are respectively the energy of the $\mathrm{Hg} 4 f_{7 / 2}$ core level fitted position as a function of time while the laser (405 nm $100 \mathrm{~mW}, 5 \mu \mathrm{s} \mathrm{50 \%} \mathrm{duty} \mathrm{cycle)} \mathrm{is} \mathrm{turned} \mathrm{on} \mathrm{and} \mathrm{off.} d$. Histogram of the on and off time of the surface photovoltage measured for a HgTe NPL film capped with EDT and $\mathrm{S}^{2-}$. The magnitudes of the error bars are based on the relative magnitude of the noise compared to the mean value of the signal.

Under illumination, we observe a shift of the binding energy associated with the $\mathrm{Hg} 4 \mathrm{f}$ core level. Figure 6 shows a shift to lower energies for EDT capped NPLs, and a positive shift for those capped with $\mathrm{S}^{2-}$. This direction of the shift further confirms the respective $p$ and $n$-type of the majority carriers for each of the ligand exchanges studied ${ }^{30}$. The turn on time is typically around $100 \mathrm{~ns}$ while the off time are longer, up to $500 \mathrm{~ns}$, as shown in Figure 6d. This time scale is typically 10 times longer than the PL decay, shown Figure 1c, because they relate to very different physical processes as discussed above. 


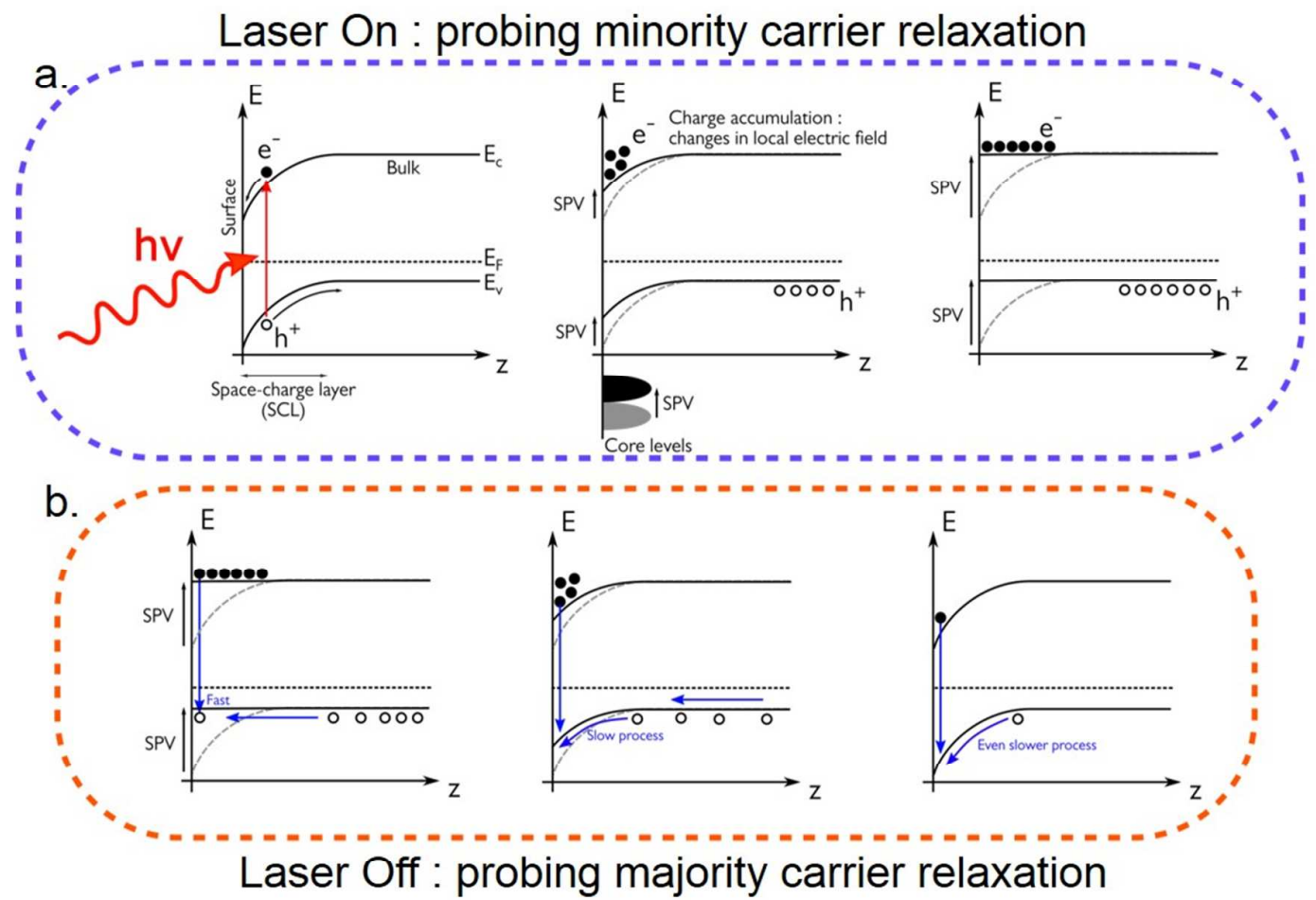

\section{c. Intra dot process}

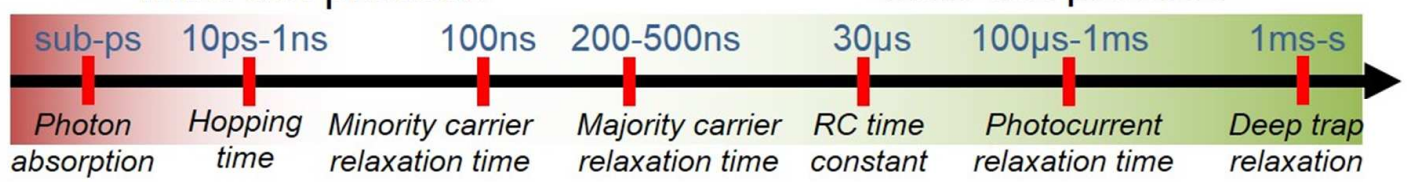

Figure 7 Scheme of the surface photovoltage process in the case of a p-doped semiconductor such as the HgTe NPL capped with EDT, when the light is turned on (part a) and when the light is turned off (part b). c. Time scale of the different carrier relaxation times in a film of HgTe NPL.

Time resolved photoemission is a surface photovoltage (SPV) measurement and provides information related to the majority and minority carrier relaxation time. During the SPV measurements, absorption occurs over the whole film, but the SPV signal (shift in binding energy associated with a change of band bending) results only from the contribution of the surface space charge region. It is worth mentioning that band bending is not occurring at the NPL scale since the thickness of the NPL is much smaller than the depletion width, which is consistent with photoemission measurement on $2 \mathrm{D} /$ layered materials ${ }^{38-40}$. Rather, we believe that the band bending takes place at the film scale. Its exact origin remains unclear but we may speculate mechanism such as a charge transfer at the substrate/NPL film interface or some thickness dependent screening of the photoemission signal ${ }^{41}$. In a doped semiconductor, the surface tends to be depleted and once photons are absorbed, excitons are generated. Due to the downward band bending occurring at the surface of a $p$-type (upward for $n$-type) semiconductor, electrons and holes are separated, and the minority carrier flows toward the surface until the establishment of a flat band (see Figure 7a). The flat band regime is reached if enough charges are photogenerated, which is confirmed in our case by the saturation of the SPV signal as we tune the pump irradiance over two orders of magnitude, see Figure S8. This flow of minority carriers is a self-decelerating process, since the driving force is the band bending and the latter is reduced by the process itself. The turn-on time, which is related to the minority carriers has been measured in the 100-150 ns range. Assuming an average value of $10^{-2} \mathrm{~cm}^{2} \mathrm{~V}^{-1} \mathrm{~s}^{-1}$ for the mobility, 
the minority carrier diffusion length associated with the SPV decay time ( $\approx 100 \mathrm{~ns})$ is given by $L_{\text {diff }} \approx \sqrt{D \tau_{S P V}}$ and the diffusion constant can be determined by the Einstein relation $D=\frac{\mu k_{b} T}{e}$, so that we obtain $L_{\text {diff }} \approx 50 \mathrm{~nm}$. This value is similar to the sample thickness ( $\approx 100 \mathrm{~nm}$ ) and has to be compared with the transit length of the photoconductive channel, which in our devices was 20 $\mu \mathrm{m}$. Thus, the difference in fall times between photocurrent and the SPV measurement can be attributed to the relevant dimension of the photoconductor being 200 times larger than that in the time-resolved photoemission measurement. This suggests that response times can be fast if the device size is similar to the diffusion length. Reducing the size of the device down to the $100 \mathrm{~nm}$ range to achieve a fast photoresponse $e^{42}$ with gain ${ }^{43-45}$ is an promising future direction for this work. It is also worth noting that sulfide capping results in a shorter SPV lifetime compared to EDT. This contributes to its weaker photoresponse, as a shorter minority carrier lifetime leads to a lower gain.

When the light is turned-off, the majority carriers now flow from the bulk of the film to refill the surface traps and restore the band bending as depicted in Figure $7 \mathrm{~b}$. The turn-off time, which is related to the majority carrier transport, is in the 200 to $500 \mathrm{~ns}$ range. This is longer than the on-time and this difference is consistent with the photoconduction measurements which similarly show decays which are slower than the rise times. Both the rise and fall times are nevertheless fast, suggesting deep traps are not involved in transport. This contrasts with observations with small $\mathrm{PbS}$ CQD, for which much longer SPV dynamics are observed ${ }^{46}$ by both time resolved photoemission ( $m s$ range) and transient photovoltage ( $\mu$ s to $\mathrm{ms}$ range). ${ }^{30}$ Indeed for small PbS CQD, traps are known to play a key role in the optoelectronic properties ${ }^{12,13}$. It is also useful to note that the measured SPV off time (majority carrier relaxation time) is below the RC time which has been measured to be around $30 \mu \mathrm{s}$ in our samples (see figure S4). This confirms that not only do HgTe NPL present better optical properties than alternative nanocrystal emitters (PbS, CulnGaS(e)) in the near IR, with narrower and faster PL, but also that the transport properties are less affected by traps. Figure 7c summarizes the different dynamics involved in this material, which appears promising for the design of photodetectors with high bandwidth (>MHz).

\section{Conclusion}

HgTe NPL appear a promising alternative to PbS CQD for near IR emission, because of their narrow emission spectrum from the lack of inhomogeneous broadening. Here we have investigated charge transport and photoresponse in this material and demonstrated that thin films of NPL can be integrated into electrolytic transistors and can be made $n$ - or $p$-type depending on their capping ligands. Photoconductivity and carrier dynamics also strongly depend on their surface chemistry. These observations were then correlated with the electronic structure determined by XPS. A strong shift of the Fermi level within the gap was observed without change of the vacuum level and in contrast to other reports with similar processing where doping resulted from dipole-induced shifts of the states. Here, doping appears to arise from hybridization which is weak with EDT and strong with sulfide. Dynamics following photoexcitation were further investigated using two complementary probes: transient photoconductivity and time resolved photoemission. The latter showed dynamics as fast as $150 \mathrm{~ns}$, which suggests that photoconductive rise and fall times coud be 3 orders faster than those we have typically observed with the device geometries used here.

\section{Acknowledgments}


We thank Francois Rochet for his comments on the photoemission data. We acknowledge the use of cleanroom facilities from the consortium "Salles Blanches Paris Centre - SBPC". We thank Agence Nationale de la Recherche for funding through grant Nanodose and $\mathrm{H} 2 \mathrm{DH}$. This work has been supported by the Region lle-de-France in the framework of DIM Nano-K. This work was supported by French state funds managed by the ANR within the Investissements d'Avenir programme under reference ANR-11-IDEX-0004-02, and more specifically within the framework of the Cluster of Excellence MATISSE.

\section{Supplementary information}

Additional data regarding the material synthesis, the transistor fabrication and the electronic structures are given in the supplementary information.

\section{REFERENCES}

(1) Lhuillier, E.; Scarafagio, M.; Hease, P.; Nadal, B.; Aubin, H.; Xu, X. Z.; Lequeux, N.; Patriarche, G.; Ithurria, S.; Dubertret, B. Nano Lett. 2016, 16, 1282-1286.

(2) Murray, C. B.; Norris, D. J.; Bawendi, M. G. J. Am. Chem. Soc. 1993, 115, 8706-8715.

(3) Klimov, V. I. Nanocrystal Quantum Dots, Second Edition; CRC Press, 2010.

(4) Talapin, D. V.; Lee, J.-S.; Kovalenko, M. V.; Shevchenko, E. V. Chem. Rev. 2010, 110, 389-458.

(5) Konstantatos and Sargent. Collidal Quantum Dot Optoelectronics and Photovoltaics; Cambridge University Press, 2013.

(6) Keuleyan, S.; Lhuillier, E.; Brajuskovic, V.; Guyot-Sionnest, P. Nat. Photonics 2011, 5, 489-493.

(7) Lhuillier, E.; Keuleyan, S.; Liu, H.; Guyot-Sionnest, P. Chem. Mater. 2013, 25, 1272-1282.

(8) Kovalenko, M. V.; Kaufmann, E.; Pachinger, D.; Roither, J.; Huber, M.; Stangl, J.; Hesser, G.; Schäffler, F.; Heiss, W. J. Am. Chem. Soc. 2006, 128, 3516-3517.

(9) Kershaw, S. V.; Susha, A. S.; Rogach, A. L. Chem. Soc. Rev. 2013, 42, 3033-3087.

(10) Lhuillier, E.; Guyot-Sionnest. IEEE J. Sel. Top. Quantum Electron. 2017, PP, 1-1.

(11) McDonald, S. A.; Konstantatos, G.; Zhang, S.; Cyr, P. W.; Klem, E. J. D.; Levina, L.; Sargent, E. H. Nat. Mater. 2005, 4, 138-142.

(12) Caram, J. R.; Bertram, S. N.; Utzat, H.; Hess, W. R.; Carr, J. A.; Bischof, T. S.; Beyler, A. P.; Wilson, M. W. B.; Bawendi, M. G. Nano Lett. 2016, 16, 6070-6077.

(13) Chuang, C.-H. M.; Maurano, A.; Brandt, R. E.; Hwang, G. W.; Jean, J.; Buonassisi, T.; Bulović, V.; Bawendi, M. G. Nano Lett. 2015, 15, 3286-3294.

(14) Nasilowski, M.; Mahler, B.; Lhuillier, E.; Ithurria, S.; Dubertret, B. Chem. Rev. 2016, 116, 10934-10982.

(15) Tessier, M. D.; Javaux, C.; Maksimovic, I.; Loriette, V.; Dubertret, B. ACS Nano 2012, 6, 67516758.

(16) Lhuillier, E.; Pedetti, S.; Ithurria, S.; Nadal, B.; Heuclin, H.; Dubertret, B. Acc. Chem. Res. 2015, 48, 22-30.

(17) Izquierdo, E.; Robin, A.; Keuleyan, S.; Lequeux, N.; Lhuillier, E.; Ithurria, S. J. Am. Chem. Soc. 2016, 138, 10496-10501.

(18) Pedetti, S.; Nadal, B.; Lhuillier, E.; Mahler, B.; Bouet, C.; Abécassis, B.; Xu, X.; Dubertret, B. Chem. Mater. 2013, 25, 2455-2462.

(19) Lhuillier, E.; Keuleyan, S.; Zolotavin, P.; Guyot-Sionnest, P. Adv. Mater. 2013, 25, 137-141.

(20) Nag, A.; Kovalenko, M. V.; Lee, J.-S.; Liu, W.; Spokoyny, B.; Talapin, D. V. J. Am. Chem. Soc. 2011, 133, 10612-10620. 
(21) Lhuillier, E.; Pedetti, S.; Ithurria, S.; Heuclin, H.; Nadal, B.; Robin, A.; Patriarche, G.; Lequeux, N.; Dubertret, B. ACS Nano 2014, 8, 3813-3820.

(22) Lhuillier, E.; Ithurria, S.; Descamps-Mandine, A.; Douillard, T.; Castaing, R.; Xu, X. Z.; Taberna, P.-L.; Simon, P.; Aubin, H.; Dubertret, B. J. Phys. Chem. C 2015, 119, 21795-21799.

(23) Lhuillier, E.; Robin, A.; Ithurria, S.; Aubin, H.; Dubertret, B. Nano Lett. 2014, 14, 2715-2719.

(24) Das, A.; Pisana, S.; Chakraborty, B.; Piscanec, S.; Saha, S. K.; Waghmare, U. V.; Novoselov, K. S.; Krishnamurthy, H. R.; Geim, A. K.; Ferrari, A. C.; Sood, A. K. Nat. Nanotechnol. 2008, 3, 210215.

(25) Robin, A.; Livache, C.; Ithurria, S.; Lacaze, E.; Dubertret, B.; Lhuillier, E. ACS Appl. Mater. Interfaces 2016, 8, 27122-27128.

(26) Brown, P. R.; Kim, D.; Lunt, R. R.; Zhao, N.; Bawendi, M. G.; Grossman, J. C.; Bulović, V. ACS Nano 2014, 8, 5863-5872.

(27) Jeong, K. S.; Deng, Z.; Keuleyan, S.; Liu, H.; Guyot-Sionnest, P. J. Phys. Chem. Lett. 2014, 5, $1139-1143$.

(28) Liu, H.; Lhuillier, E.; Guyot-Sionnest, P. J. Appl. Phys. 2014, 115, 154309.

(29) Nimtz, G.; Schlicht, B.; Dornhaus, R. Narrow-gap semiconductors; Springer; 1983.

(30) Lin, W. M. M.; Bozyigit, D.; Yarema, O.; Wood, V. J. Phys. Chem. C 2016, 120, 12900-12908.

(31) Rabouw, F. T.; Donega, C. de M. Top. Curr. Chem. 2016, 374, 58.

(32) Cassette, E.; Dean, J. C.; Scholes, G. D. Small 2016, 12, 2234-2244.

(33) Gao, J.; Fidler, A. F.; Klimov, V. I. Nat. Commun. 2015, 6.

(34) Pietryga, J. M.; Park, Y.-S.; Lim, J.; Fidler, A. F.; Bae, W. K.; Brovelli, S.; Klimov, V. I. Chem. Rev. 2016, 116, 10513-10622.

(35) Keuleyan, S.; Kohler, J.; Guyot-Sionnest, P. J. Phys. Chem. C 2014, 118, 2749-2753.

(36) Spencer, B. F.; Cliffe, M. J.; Graham, D. M.; Hardman, S. J. O.; Seddon, E. A.; Syres, K. L.; Thomas, A. G.; Sirotti, F.; Silly, M. G.; Akhtar, J.; O’Brien, P.; Fairclough, S. M.; Smith, J. M.; Chattopadhay, S.; Flavell, W. R. Surf. Sci. 2015, 641, 320-325.

(37) Spencer, B. F.; Graham, D. M.; Hardman, S. J. O.; Seddon, E. A.; Cliffe, M. J.; Syres, K. L.; Thomas, A. G.; Stubbs, S. K.; Sirotti, F.; Silly, M. G.; Kirkham, P. F.; Kumarasinghe, A. R.; Hirst, G. J.; Moss, A. J.; Hill, S. F.; Shaw, D. A.; Chattopadhyay, S.; Flavell, W. R. Phys. Rev. B 2013, 88, 195301.

(38) Chiu, M. H.; Zhang, C.; Shiu, H. W.; Chuu, C. P.; Chen, C. H.; Chang, C. Y.; Chen, C. H.; Chou, M. Y.; Shih, C. K.; Li, L. J. Nat. Commun. 2015, 6, 7666.

(39) Pierucci, D.; Henck, H.; Avila, J.; Balan, A.; Naylor, C. H.; Patriarche, G.; Dappe, Y. J.; Silly, M. G.; Sirotti, F.; Johnson, A. T. C.; Asensio, M. C.; Ouerghi, A. Nano Lett. 2016, 16, 4054-4061.

(40) Lang, O.; Klein, A.; Pettenkofer, C.; Jaegermann, W.; Chevy, A. J. Appl. Phys. 1998.

(41) Helander, M. G.; Greiner, M. T.; Wang, Z. B.; Lu, Z. H. Phys. Rev. B 2010, 81, 153308.

(42) Gao, J.; Nguyen, S. C.; Bronstein, N. D.; Alivisatos, A. P. ACS Photonics 2016, 3, 1217-1222.

(43) Lhuillier, E.; Dayen, J.-F.; Thomas, D. O.; Robin, A.; Doudin, B.; Dubertret, B. Nano Lett. 2015, 15, 1736-1742.

(44) Wang, H.; Lhuillier, E.; Yu, Q.; Zimmers, A.; Dubertret, B.; Ulysse, C.; Aubin, H. ACS Nano 2017, $11,1222-1229$.

(45) Prins, F.; Buscema, M.; Seldenthuis, J. S.; Etaki, S.; Buchs, G.; Barkelid, M.; Zwiller, V.; Gao, Y.; Houtepen, A. J.; Siebbeles, L. D. A.; van der Zant, H. S. J. Nano Lett. 2012, 12, 5740-5743.

(46) Spencer, B. F.; Leontiadou, M. A.; Clark, P. C. J.; Williamson, A. I.; Silly, M. G.; Sirotti, F.; Fairclough, S. M.; Tsang, S. C. E.; Neo, D. C. J.; Assender, H. E.; Watt, A. a. R.; Flavell, W. R. Appl. Phys. Lett. 2016, 108, 91603. 
Table of content graphic

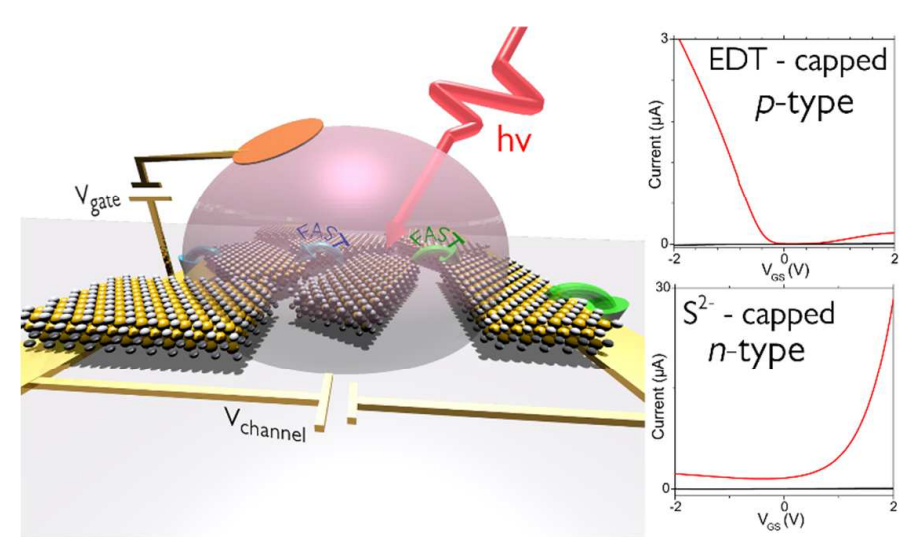

